\title{
Streptococcus pneumoniae Vaccine
}

National Cancer Institute

\section{Source}

National Cancer Institute. Streptococcus pneumoniae Vaccine. NCI Thesaurus. Code C96393.

A bacterial vaccine used to prevent contraction of Streptococcus pneumonia. 